CRYSTALLOGRAPHIC COMMUNICATIONS

ISSN 2056-9890

Received 8 September 2017

Accepted 19 September 2017

Edited by W. Imhof, University Koblenz-Landau, Germany

Keywords: crystal structure; ferrocene; amines; phosphines; structure elucidation.

CCDC reference: 1575391

Supporting information: this article has supporting information at journals.iucr.org/e

\section{Crystal structure of $\left\{\left[1^{\prime}\right.\right.$-(diphenylphosphino)- ferrocenyl]methyl\}dimethylammonium chloride monohydrate}

\author{
Martin Zábranský and Petr Štěpnička*
}

Department of Inorganic Chemistry, Faculty of Science, Charles University, Hlavova 2030, 12840 Prague 2, Czech Republic. *Correspondence e-mail: petr.stepnicka@natur.cuni.cz

Individual ions and the solvating water molecule constituting the structure of the title compound, $\left[\mathrm{Fe}\left(\mathrm{C}_{8} \mathrm{H}_{13} \mathrm{~N}\right)\left(\mathrm{C}_{17} \mathrm{H}_{14} \mathrm{P}\right)\right] \mathrm{Cl} \cdot \mathrm{H}_{2} \mathrm{O}$, assemble into dimeric units located around crystallographic inversion centers via $\mathrm{N}-\mathrm{H} \cdots \mathrm{Cl}$ and $\mathrm{O}-$ $\mathrm{H}$. . Cl hydrogen bonds. These discrete fragments are further interconnected into chains by $\mathrm{C}-\mathrm{H} \cdots \mathrm{O}$ interactions. The disubstituted ferrocene core in the $\left\{\left[1^{\prime}\right.\right.$-(diphenylphosphino)ferrocenyl $]$ methyl $\}$ dimethylammonium cation has an approximate synclinal eclipsed conformation and is tilted by $3.40(11)^{\circ}$.

\section{Chemical context}

Chiral phosphinoferrocene amines are recognized to be efficient supporting ligands for transition-metal-catalysed reactions as well as useful synthetic precursors for a range of ferrocene derivatives (Štěpnička et al., 2008). In contrast, their non-chiral counterparts have received limited attention. While studying functional derivatives of the ubiquitous 1,1'-bis(diphenylphosphino)ferrocene (dppf), we have devised an alternative synthesis of $1^{\prime}$-(diphenylphosphino)-1-[(dimethylamino)methyl]ferrocene, $\mathrm{Ph}_{2} \mathrm{PfcCH}_{2} \mathrm{NMe}_{2}$ (fc = ferrocene$1,1^{\prime}$-diyl), firstly reported by Wright (1990), and studied this compound as a ligand in $\mathrm{Pd}^{\mathrm{II}}$ and $\mathrm{Au}^{\mathrm{I}}$ complexes (Štěpnička et al., 2012). More recently, we have converted this phosphinoamine into a phosphinoferrocene betaine $\mathrm{Ph}_{2} \mathrm{PfcCH}_{2} \mathrm{NMe}_{2}\left(\mathrm{CH}_{2}\right)_{3} \mathrm{SO}_{3}$, which was in turn used to prepare new functional ferrocene phosphines (Zábranský et al., 2015, 2017). This contribution describes the crystal structure of a hydrated hydrochloride of this amine, $\left[\mathrm{Ph}_{2} \mathrm{PfcCH}_{2} \mathrm{NHMe}_{2}\right] \mathrm{Cl} \cdot \mathrm{H}_{2} \mathrm{O}$, which was isolated serendipitously while regenerating the amine after preparation of the aforementioned betaine.

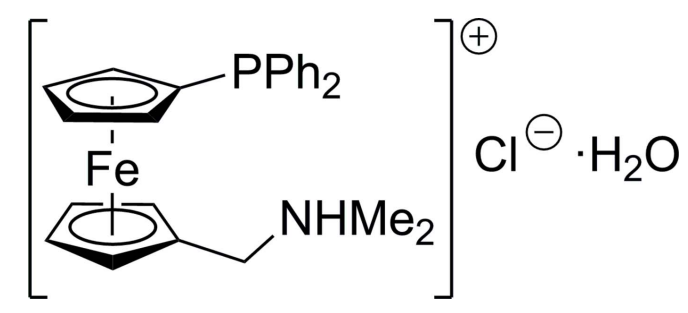

\section{Structural commentary}

A view of the molecular structure of the title compound, with atom labelling, is shown in Fig. 1. The ferrocene moiety in 


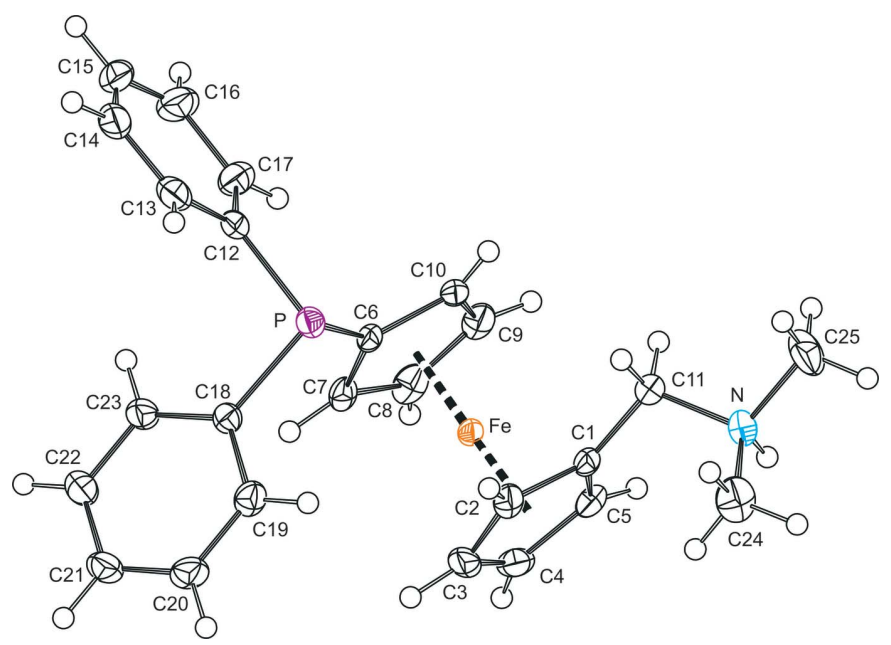

Figure 1

PLATON (Spek, 2009) plot of the cation in the structure of the title compound. Displacement ellipsoids correspond to the $50 \%$ probability level.

the $\left\{\left[1^{\prime}\right.\right.$-(diphenylphosphino)ferrocenyl $]$ methyl $\}$ dimethylammonium cation has a regular geometry with the individual $\mathrm{Fe}-\mathrm{C}$ bonds ranging from 2.0239 (15) $\AA$ (C1) to 2.0489 (15) $\AA$ (C7). Its cyclopentadienyl rings are tilted by $3.40(11)^{\circ}$ and assume an eclipsed conformation with the attached substituents oriented in a synclinal fashion, as demonstrated by the torsion angle $\mathrm{C} 1-C g 1-C g 2-\mathrm{C} 6$ of $-85.38(12)^{\circ}$, where $C g 1$ and $C g 2$ are the centroids of the cyclopentadienyl rings $\mathrm{C} 1-\mathrm{C} 5$ and $\mathrm{C} 6-\mathrm{C} 10$, respectively.

The protonated aminomethyl chain is directed away from the ferrocene core, with the angle between the $\mathrm{C} 1-\mathrm{N}$ bond and the axis of the ferrocene unit, $C g 1 \cdots C g 2$, being $148.99(11)^{\circ}$. The phosphine substituent at the other cyclopentadienyl ring is oriented so that one of its pivotal $\mathrm{P}-\mathrm{C}(\mathrm{Ph})$ bonds lies nearly in the plane of the bonding five-membered

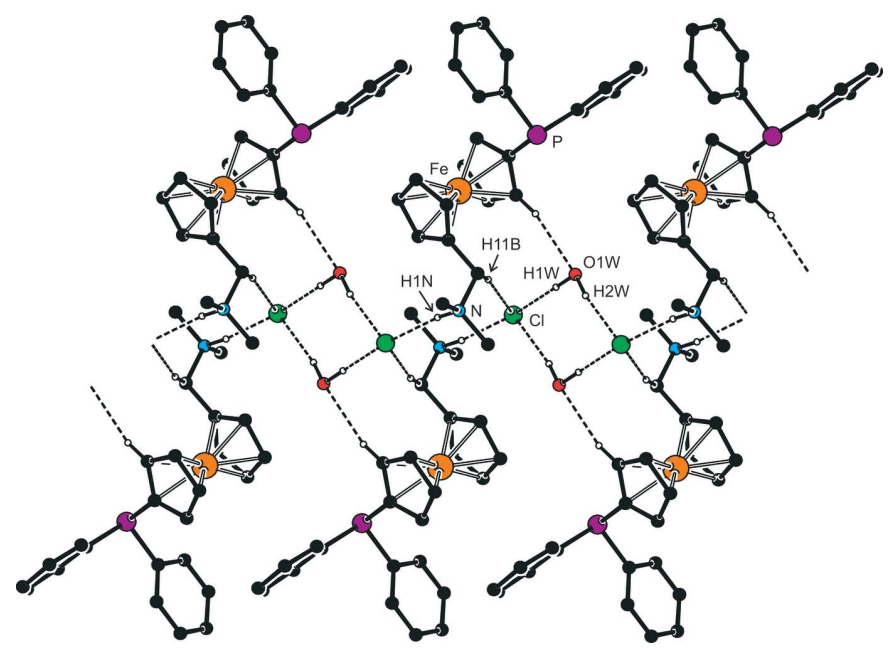

Figure 2

Section of the hydrogen-bonded chains in the structure of the title compound. For clarity, hydrogen atoms not involved in hydrogen bonding are omitted. ring $\mathrm{C} 6-\mathrm{C} 10$, while the other is roughly parallel with the axis of the ferrocene unit. The angle at which the $\mathrm{P}-\mathrm{C} 18$ bond intersects the $\mathrm{C} 6-\mathrm{C} 10$ plane is $13.17(10)^{\circ}$, whereas the angle subtended by the $\mathrm{P}-\mathrm{C} 12$ bond and the $C g 1 \cdots C g 2$ line is only $8.68(5)^{\circ}$.

\section{Supramolecular features}

Each $\left[\mathrm{Ph}_{2} \mathrm{PfcCH}_{2} \mathrm{NHMe}_{2}\right]^{+}$cation in the structure of the title compound is involved in an $\mathrm{N}-\mathrm{H} \cdots \mathrm{Cl}$ hydrogen bond to a proximal chloride anion (for hydrogen-bond parameters, see Table 1). The anions further act as hydrogen-bond acceptors for a pair of inversion-related water molecules, which in turn results in the formation of charge-neutral, closed dimeric arrays $\left\{\left(\mathrm{Ph}_{2} \mathrm{PfcCH}_{2} \mathrm{NHMe}_{2}\right)_{2} \mathrm{Cl}_{2}\left(\mathrm{H}_{2} \mathrm{O}\right)_{2}\right\}$ around the crystallographic inversion centers. These discrete units are further interlinked into chains along the $a$ axis via the weaker $\mathrm{C}-$ $\mathrm{H} \cdots \mathrm{O}$ and $\mathrm{C}-\mathrm{H} \cdots \mathrm{Cl}$ interactions, as shown in Fig. 2.

\section{Database survey}

A search in the Cambridge Structural Database (Version 5.38 with the latest update from May 2017; Groom et al., 2016) for structurally related compounds resulted in the structures of two similar (ferrocenylmethyl)ammonium salts, namely $N$-(ferrocenylmethyl)dimethylammonium chloride (Winter \& Wolmershäuser, 1998) and its dihydrate (Guo et al., 2006), and two complexes obtained from $\mathrm{Ph}_{2} \mathrm{PfcCH}_{2} \mathrm{NMe}_{2}$ featuring a protonated (dimethylamino)methyl side chain, viz. $\left[\mathrm{AuCl}\left(\mathrm{Ph}_{2} \mathrm{PfcCH}_{2} \mathrm{NHMe}_{2}\right)\right] X$, where $X=\mathrm{Cl}$ and $\mathrm{ClO}_{4}$ (Štěpnička et al., 2012).

\section{Synthesis and crystallization}

The 'amine' $\mathrm{Ph}_{2} \mathrm{PfcCH}_{2} \mathrm{NMe}_{2}$ regenerated from the synthesis of the phosphinoferrocene betaine $\mathrm{Ph}_{2} \mathrm{PfcCH}_{2} \mathrm{NMe}_{2}$ $\left(\mathrm{CH}_{2}\right)_{3} \mathrm{SO}_{3}$ (Zábranský et al., 2015) (ca $100 \mathrm{mg}$ ) was dissolved in acetic acid $(10 \mathrm{~mL})$ and the solution was evaporated under reduced pressure. After this procedure was repeated twice using chloroform as a solvent, the residue was dissolved in a minimum amount of hot ethyl acetate. The solution was filtered and layered with hexane. Crystallization by liquidphase diffusion over several days afforded orange crystals of the title compound. The yield was not determined.

Analysis calculated for $\left[\mathrm{C}_{25} \mathrm{H}_{27} \mathrm{FeNP}\right] \mathrm{Cl} \cdot \mathrm{H}_{2} \mathrm{O}$ (481.76 $\mathrm{g} \mathrm{mol}^{-1}$ ): C 62.32, H 6.07, N 2.91\%. Found: C 62.23, H

Table 1

Hydrogen-bond geometry $\left(\AA{ }^{\circ}\right)$.

\begin{tabular}{lllll}
\hline$D-\mathrm{H} \cdots A$ & $D-\mathrm{H}$ & $\mathrm{H} \cdots A$ & $D \cdots A$ & $D-\mathrm{H} \cdots A$ \\
\hline $\mathrm{N}-\mathrm{H} 1 N \cdots \mathrm{Cl}$ & 0.92 & 2.13 & $3.0323(16)$ & 167 \\
$\mathrm{O} 1 W-\mathrm{H} 1 W \cdots \mathrm{Cl}$ & 0.98 & 2.23 & $3.2162(19)$ & 177 \\
$\mathrm{O} 1 W-\mathrm{H} 2 W \cdots \mathrm{Cl}^{\mathrm{i}}$ & 1.02 & 2.29 & $3.289(2)$ & 166 \\
$\mathrm{C} 10-\mathrm{H} 10 \cdots \mathrm{O} 1 W^{\mathrm{ii}}$ & 0.95 & 2.46 & $3.390(3)$ & 165 \\
$\mathrm{C} 11-\mathrm{H} 11 B \cdots \mathrm{Cl}^{\mathrm{ii}}$ & 0.99 & 2.77 & $3.7369(17)$ & 167 \\
\hline
\end{tabular}

Symmetry codes: (i) $-x,-y+2,-z+2$; (ii) $-x+1,-y+2,-z+2$. 
Table 2

Experimental details.

\begin{tabular}{|c|c|}
\hline \multicolumn{2}{|l|}{ Crystal data } \\
\hline Chemical formula & {$\left[\mathrm{Fe}\left(\mathrm{C}_{8} \mathrm{H}_{13} \mathrm{~N}\right)\left(\mathrm{C}_{17} \mathrm{H}_{14} \mathrm{P}\right)\right] \mathrm{Cl} \cdot \mathrm{H}_{2} \mathrm{O}$} \\
\hline$M_{\mathrm{r}}$ & 481.76 \\
\hline Crystal system, space group & Triclinic, $P \overline{1}$ \\
\hline Temperature (K) & 150 \\
\hline$a, b, c(\AA)$ & $7.9888(3), 12.7596(6), 13.2311(5)$ \\
\hline$\alpha, \beta, \gamma\left({ }^{\circ}\right)$ & 111.037 (1), $104.075(1), 99.628(2)$ \\
\hline$V\left(\AA^{3}\right)$ & $1171.76(8)$ \\
\hline$Z$ & 2 \\
\hline Radiation type & Мо $K \alpha$ \\
\hline$\mu\left(\mathrm{mm}^{-1}\right)$ & 0.84 \\
\hline Crystal size (mm) & $0.27 \times 0.26 \times 0.14$ \\
\hline \multicolumn{2}{|l|}{ Data collection } \\
\hline Diffractometer & $\begin{array}{c}\text { Bruker D8 VENTURE Kappa } \\
\text { Duo PHOTON } 100 \text { CMOS }\end{array}$ \\
\hline Absorption correction & $\begin{array}{l}\text { Numerical (SADABS; Bruker, } \\
\text { 2014) }\end{array}$ \\
\hline$T_{\min }, T_{\max }$ & $0.78,0.89$ \\
\hline $\begin{array}{l}\text { No. of measured, independent and } \\
\text { observed }[I>2 \sigma(I)] \text { reflections }\end{array}$ & $24764,5361,4820$ \\
\hline$R_{\text {int }}$ & 0.024 \\
\hline$(\sin \theta / \lambda)_{\max }\left(\AA^{-1}\right)$ & 0.650 \\
\hline \multicolumn{2}{|l|}{ Refinement } \\
\hline$R\left[F^{2}>2 \sigma\left(F^{2}\right)\right], w R\left(F^{2}\right), S$ & $0.028,0.074,1.06$ \\
\hline No. of reflections & 5361 \\
\hline No. of parameters & 273 \\
\hline $\mathrm{H}$-atom treatment & $\mathrm{H}$-atom parameters constrained \\
\hline$\Delta \rho_{\max }, \Delta \rho_{\min }\left(\mathrm{e} \AA^{-3}\right)$ & $0.77,-0.53$ \\
\hline
\end{tabular}

Computer programs: Instrument Service and SAINT (Bruker, 2015), SHELXT (Sheldrick, 2015a), SHELXL2014 (Sheldrick, 2015b) and PLATON (Spek, 2009).

5.91, N 2.79\%. ESI MS: $m / z \quad 383\left(\left[\mathrm{Ph}_{2} \mathrm{PfcCH}_{2}\right]^{+}\right), 428$ $\left(\left[\mathrm{Ph}_{2} \mathrm{PfcCH}_{2} \mathrm{NMe}_{2}+\mathrm{H}\right]^{+}\right)$

\section{Refinement}

Relevant crystallographic data and structure refinement parameters are summarized in Table 2. All non-hydrogen atoms were refined freely with anisotropic displacement parameters. The hydrogen atoms of the water molecule and the NH proton were located on a difference electron-density map and refined as riding atoms with $U_{\text {iso }}(\mathrm{H})$ set to $1.2 U_{\text {eq }}$ of their bonding atom. Hydrogen atoms bonded to carbons were included in their theoretical positions and refined as riding atoms with $U_{\text {iso }}(\mathrm{H})=1.2 U_{\text {eq }}(\mathrm{C})$.

\section{Acknowledgements}

The authors are grateful to Dr Ivana Císařová from the Department of Inorganic Chemistry, Faculty of Science, Charles University for recording the diffraction data of the title compound.

\section{Funding information}

This work was supported by the Czech Science Foundation (project no. 15-11571S).

\section{References}

Bruker (2014). SADABS. Bruker AXS Inc., Madison, Wisconsin, USA.

Bruker (2015). Instrument Service and SAINT. Bruker AXS Inc., Madison, Wisconsin, USA.

Groom, C. R., Bruno, I. J., Lightfoot, M. P. \& Ward, S. C. (2016). Acta Cryst. B72, 171-179.

Guo, H.-X., Zhou, X.-J., Lin, Z.-X. \& Liu, J.-M. (2006). Acta Cryst. E62, m1770-m1772.

Sheldrick, G. M. (2015a). Acta Cryst. A71, 3-8.

Sheldrick, G. M. (2015b). Acta Cryst. C71, 3-8.

Spek, A. L. (2009). Acta Cryst. D65, 148-155.

Štěpnička, P. (2008). Ferrocenes: Ligands, Materials and Biomolecules. Chichester: Wiley.

Štěpnička, P., Zábranský, M. \& Císařová, I. (2012). ChemistryOpen 1, pp. 71-79.

Winter, R. F. \& Wolmershäuser, G. (1998). J. Organomet. Chem. 570, 201-218.

Wright, M. E. (1990). Organometallics, 9, 853-856.

Zábranský, M., Císařová, I. \& Štěpnička, P. (2015). Dalton Trans. 44, 14494-14506.

Zábranský, M., Císařová, I. \& Štěpnička, P. (2017). Eur. J. Inorg. Chem. pp. 2557-2572. 


\section{supporting information}

Acta Cryst. (2017). E73, 1539-1541 [https://doi.org/10.1107/S2056989017013408]

\section{Crystal structure of \{[1'-(diphenylphosphino)ferrocenyl] methyl\}dimethyl- ammonium chloride monohydrate}

Martin Zábranský and Petr Štěpnička

Computing details

Data collection: Instrument Service (Bruker, 2015); cell refinement: SAINT (Bruker, 2015); data reduction: SAINT

(Bruker, 2015); program(s) used to solve structure: SHELXT (Sheldrick, 2015a); program(s) used to refine structure: SHELXL2014 (Sheldrick, 2015b); molecular graphics: PLATON (Spek, 2009); software used to prepare material for publication: PLATON (Spek, 2009).

$\{[1 '$-(Diphenylphosphino)ferrocenyl] methyl\}dimethylammonium chloride monohydrate

\section{Crystal data}

$\left[\mathrm{Fe}\left(\mathrm{C}_{8} \mathrm{H}_{13} \mathrm{~N}\right)\left(\mathrm{C}_{17} \mathrm{H}_{14} \mathrm{P}\right] \mathrm{Cl} \cdot \mathrm{H}_{2} \mathrm{O}\right.$

$M_{r}=481.76$

Triclinic, $P \overline{1}$

$a=7.9888(3) \AA$

$b=12.7596(6) \AA$

$c=13.2311(5) \AA$

$\alpha=111.037(1)^{\circ}$

$\beta=104.075(1)^{\circ}$

$\gamma=99.628(2)^{\circ}$

$V=1171.76(8) \AA^{3}$

\section{Data collection}

Bruker D8 VENTURE Kappa Duo PHOTON 100 CMOS diffractometer

Radiation source: $\mathrm{I} \mu \mathrm{S}$ micro-focus sealed tube Quazar Mo multilayer optic monochromator $\varphi$ and $\omega$ scans

Absorption correction: numerical

(SADABS; Bruker, 2014)

$T_{\min }=0.78, T_{\max }=0.89$

\section{Refinement}

Refinement on $F^{2}$

Least-squares matrix: full

$R\left[F^{2}>2 \sigma\left(F^{2}\right)\right]=0.028$

$w R\left(F^{2}\right)=0.074$

$S=1.06$

5361 reflections

273 parameters

0 restraints

\section{$Z=2$}

$F(000)=504$

$D_{\mathrm{x}}=1.365 \mathrm{Mg} \mathrm{m}^{-3}$

Mo $K \alpha$ radiation, $\lambda=0.71073 \AA$

Cell parameters from 9875 reflections

$\theta=2.7-27.5^{\circ}$

$\mu=0.84 \mathrm{~mm}^{-1}$

$T=150 \mathrm{~K}$

Plate, orange

$0.27 \times 0.26 \times 0.14 \mathrm{~mm}$

24764 measured reflections

5361 independent reflections

4820 reflections with $I>2 \sigma(I)$

$R_{\text {int }}=0.024$

$\theta_{\max }=27.5^{\circ}, \theta_{\min }=2.7^{\circ}$

$h=-10 \rightarrow 10$

$k=-16 \rightarrow 16$

$l=-17 \rightarrow 15$

Primary atom site location: structure-invariant direct methods

Secondary atom site location: difference Fourier map

Hydrogen site location: mixed

$\mathrm{H}$-atom parameters constrained

$w=1 /\left[\sigma^{2}\left(F_{\mathrm{o}}{ }^{2}\right)+(0.0302 P)^{2}+0.8395 P\right]$

where $P=\left(F_{\mathrm{o}}{ }^{2}+2 F_{\mathrm{c}}{ }^{2}\right) / 3$ 
$(\Delta / \sigma)_{\max }<0.001$

$\Delta \rho_{\max }=0.77$ e $\AA^{-3}$

$\Delta \rho_{\min }=-0.53$ e $\AA^{-3}$

Special details

Geometry. All esds (except the esd in the dihedral angle between two 1.s. planes) are estimated using the full covariance matrix. The cell esds are taken into account individually in the estimation of esds in distances, angles and torsion angles; correlations between esds in cell parameters are only used when they are defined by crystal symmetry. An approximate (isotropic) treatment of cell esds is used for estimating esds involving l.s. planes.

Fractional atomic coordinates and isotropic or equivalent isotropic displacement parameters $\left(\hat{A}^{2}\right)$

\begin{tabular}{|c|c|c|c|c|}
\hline & $x$ & $y$ & $z$ & $U_{\text {iso }} * / U_{\text {eq }}$ \\
\hline $\mathrm{Fe}$ & 0.35629 & $0.59451(2)$ & $0.78521(2)$ & $0.01686(7)$ \\
\hline $\mathrm{P}$ & $0.60918(5)$ & 0.42728 & $0.65913(3)$ & $0.02008(9)$ \\
\hline $\mathrm{N}$ & 0.48959 (19) & $0.94040(11)$ & $0.77917(12)$ & $0.0222(3)$ \\
\hline $\mathrm{H} 1 \mathrm{~N}$ & 0.4115 & 0.9619 & 0.8185 & $0.027^{*}$ \\
\hline $\mathrm{C} 1$ & $0.3623(2)$ & $0.73548(13)$ & $0.74808(13)$ & $0.0190(3)$ \\
\hline $\mathrm{C} 2$ & $0.3012(2)$ & $0.63030(14)$ & $0.64396(13)$ & $0.0246(3)$ \\
\hline $\mathrm{H} 2$ & 0.3559 & 0.6120 & 0.5860 & $0.029 *$ \\
\hline $\mathrm{C} 3$ & $0.1436(2)$ & $0.55845(15)$ & $0.64331(15)$ & $0.0305(4)$ \\
\hline H3 & 0.0755 & 0.4832 & 0.5850 & $0.037 *$ \\
\hline $\mathrm{C} 4$ & $0.1056(2)$ & $0.61835(16)$ & $0.74453(17)$ & $0.0306(4)$ \\
\hline $\mathrm{H} 4$ & 0.0075 & 0.5903 & 0.7655 & $0.037 *$ \\
\hline $\mathrm{C} 5$ & $0.2396(2)$ & $0.72753(15)$ & $0.80907(15)$ & $0.0242(3)$ \\
\hline H5 & 0.2463 & 0.7853 & 0.8805 & $0.029 *$ \\
\hline C6 & $0.5294(2)$ & $0.49253(13)$ & 0.77727 (13) & $0.0188(3)$ \\
\hline $\mathrm{C} 7$ & $0.3773(2)$ & $0.44264(14)$ & $0.80049(13)$ & $0.0214(3)$ \\
\hline $\mathrm{H} 7$ & 0.2959 & 0.3668 & 0.7549 & $0.026^{*}$ \\
\hline $\mathrm{C} 8$ & $0.3684(3)$ & $0.52571(16)$ & $0.90340(14)$ & $0.0282(4)$ \\
\hline $\mathrm{H} 8$ & 0.2794 & 0.5153 & 0.9380 & $0.034 *$ \\
\hline C9 & 0.5155 & $0.62697(15)$ & $0.94565(14)$ & $0.0295(4)$ \\
\hline H9 & 0.5427 & 0.6956 & 1.0139 & $0.035 *$ \\
\hline $\mathrm{C} 10$ & $0.6153(2)$ & $0.60790(14)$ & $0.86822(14)$ & 0.0240 \\
\hline $\mathrm{H} 10$ & 0.7199 & 0.6617 & 0.8753 & $0.029 *$ \\
\hline $\mathrm{C} 11$ & $0.5289(2)$ & $0.83286(14)$ & $0.79066(14)$ & $0.0231(3)$ \\
\hline H11A & 0.6098 & 0.8067 & 0.7468 & $0.028 *$ \\
\hline H11B & 0.5921 & 0.8529 & 0.8723 & $0.028 *$ \\
\hline $\mathrm{C} 12$ & $0.7610(2)$ & $0.35513(13)$ & $0.71926(14)$ & $0.0218(3)$ \\
\hline $\mathrm{C} 13$ & $0.8462(2)$ & $0.28984(15)$ & 0.64905 (17) & $0.0315(4)$ \\
\hline H13 & 0.8258 & 0.2852 & 0.5736 & $0.038 *$ \\
\hline $\mathrm{C} 14$ & $0.9599(2)$ & $0.23210(16)$ & $0.6890(2)$ & $0.0422(5)$ \\
\hline H14 & 1.0132 & 0.1856 & 0.6396 & $0.051 *$ \\
\hline $\mathrm{C} 15$ & $0.9964(3)$ & $0.24150(17)$ & $0.7998(2)$ & $0.0442(6)$ \\
\hline H15 & 1.0754 & 0.2022 & 0.8268 & $0.053 *$ \\
\hline $\mathrm{C} 16$ & 0.9178 & $0.30812(18)$ & $0.8711(2)$ & $0.0395(5)$ \\
\hline H16 & 0.9444 & 0.3160 & 0.9479 & $0.047 *$ \\
\hline $\mathrm{C} 17$ & $0.7991(2)$ & $0.36410(16)$ & $0.83049(16)$ & $0.0290(4)$ \\
\hline H17 & 0.7439 & 0.4088 & 0.8797 & $0.035^{*}$ \\
\hline
\end{tabular}




$\begin{array}{lllll}\mathrm{C} 18 & 0.4138(2) & 0.30073(13) & 0.56095(13) & 0.0194(3) \\ \mathrm{C} 19 & 0.2952(2) & 0.31205(15) & 0.47127(14) & 0.0257(3) \\ \mathrm{H} 19 & 0.3154 & 0.3842 & 0.4641 & 0.031^{*} \\ \mathrm{C} 20 & 0.1473(2) & 0.21872(16) & 0.39223(15) & 0.0303(4) \\ \mathrm{H} 20 & 0.0669 & 0.2278 & 0.3321 & 0.036^{*} \\ \mathrm{C} 21 & 0.1172(2) & 0.11288(16) & 0.40097(15) & 0.0293(4) \\ \mathrm{H} 21 & 0.0170 & 0.0491 & 0.3465 & 0.035^{*} \\ \mathrm{C} 22 & 0.2337(2) & 0.10042(15) & 0.48951(15) & 0.0298(4) \\ \mathrm{H} 22 & 0.2135 & 0.0278 & 0.4957 & 0.036^{*} \\ \mathrm{C} 23 & 0.3807(2) & 0.19393(14) & 0.56956(14) & 0.0249(3) \\ \mathrm{H} 23 & 0.4590 & 0.1848 & 0.6306 & 0.030^{*} \\ \mathrm{C} 24 & 0.3962(3) & 0.91775(17) & 0.65899(16) & 0.0363(4) \\ \mathrm{H} 24 \mathrm{~A} & 0.3688 & 0.9890 & 0.6563 & 0.054^{*} \\ \mathrm{H} 24 \mathrm{~B} & 0.2841 & 0.8547 & 0.6282 & 0.054^{*} \\ \mathrm{H} 24 \mathrm{C} & 0.4738 & 0.8945 & 0.6128 & 0.054^{*} \\ \mathrm{C} 25 & 0.6568(3) & 1.03899(16) & 0.83233(19) & 0.0405(5) \\ \mathrm{H} 25 \mathrm{~A} & 0.7412 & 1.0191 & 0.7907 & 0.061^{*} \\ \mathrm{H} 25 \mathrm{~B} & 0.7117 & 1.0534 & 0.9126 & 0.061^{*} \\ \mathrm{H} 25 \mathrm{C} & 0.6277 & 1.1095 & 0.8288 & 0.061^{*} \\ \mathrm{Cl} & 0.23009(6) & 1.03869(4) & 0.89357(4) & 0.02978(10) \\ \text { O1W } & 0.0368(2) & 1.16218(15) & 1.07177(16) & 0.0569(4) \\ \mathrm{H} 2 \mathrm{~W} & -0.0268 & 1.1016 & 1.0938 & 0.068^{*} \\ \mathrm{H} 1 \mathrm{~W} & 0.0973 & 1.1233 & 1.0189 & 0.068^{*} \\ & & & & \end{array}$

Atomic displacement parameters $\left(\AA^{2}\right)$

\begin{tabular}{lllllll}
\hline & $U^{11}$ & $U^{22}$ & $U^{33}$ & $U^{12}$ & $U^{13}$ & $U^{23}$ \\
\hline $\mathrm{Fe}$ & $0.01965(11)$ & $0.01487(11)$ & $0.01374(11)$ & $0.00601(8)$ & $0.00136(8)$ & $0.00560(8)$ \\
$\mathrm{P}$ & $0.0215(2)$ & $0.01765(19)$ & $0.01958(19)$ & $0.00385(15)$ & $0.00531(15)$ & $0.00777(15)$ \\
$\mathrm{N}$ & $0.0266(7)$ & $0.0174(6)$ & $0.0224(7)$ & $0.0044(5)$ & $0.0097(6)$ & $0.0076(5)$ \\
$\mathrm{C} 1$ & $0.0216(7)$ & $0.0165(7)$ & $0.0189(7)$ & $0.0076(6)$ & $0.0027(6)$ & $0.0089(6)$ \\
$\mathrm{C} 2$ & $0.0342(9)$ & $0.0206(8)$ & $0.0164(7)$ & $0.0094(7)$ & $0.0009(6)$ & $0.0092(6)$ \\
$\mathrm{C} 3$ & $0.0296(9)$ & $0.0221(8)$ & $0.0272(8)$ & $0.0013(7)$ & $-0.0102(7)$ & $0.0117(7)$ \\
$\mathrm{C} 4$ & $0.0178(8)$ & $0.0319(9)$ & $0.0445(10)$ & $0.0069(7)$ & $0.0042(7)$ & $0.0225(8)$ \\
$\mathrm{C} 5$ & $0.0243(8)$ & $0.0242(8)$ & $0.0298(8)$ & $0.0141(7)$ & $0.0101(7)$ & $0.0137(7)$ \\
$\mathrm{C} 6$ & $0.0212(7)$ & $0.0152(7)$ & $0.0183(7)$ & $0.0073(6)$ & $0.0021(6)$ & $0.0071(6)$ \\
$\mathrm{C} 7$ & $0.0282(8)$ & $0.0188(7)$ & $0.0214(7)$ & $0.0090(6)$ & $0.0078(6)$ & $0.0122(6)$ \\
$\mathrm{C} 8$ & $0.0408(10)$ & $0.0328(9)$ & $0.0202(8)$ & $0.0186(8)$ & $0.0127(7)$ & $0.0160(7)$ \\
$\mathrm{C} 9$ & $0.0409(10)$ & $0.0271(9)$ & $0.0147(7)$ & $0.0170(8)$ & $-0.0003(7)$ & $0.0052(6)$ \\
$\mathrm{C} 10$ & $0.0226(8)$ & $0.0178(7)$ & $0.0222(8)$ & $0.0067(6)$ & $-0.0048(6)$ & $0.0056(6)$ \\
$\mathrm{C} 11$ & $0.0216(8)$ & $0.0201(7)$ & $0.0261(8)$ & $0.0070(6)$ & $0.0051(6)$ & $0.0091(6)$ \\
$\mathrm{C} 12$ & $0.0160(7)$ & $0.0162(7)$ & $0.0273(8)$ & $0.0016(6)$ & $0.0043(6)$ & $0.0059(6)$ \\
$\mathrm{C} 13$ & $0.0202(8)$ & $0.0249(8)$ & $0.0353(10)$ & $0.0023(7)$ & $0.0078(7)$ & $-0.0001(7)$ \\
$\mathrm{C} 14$ & $0.0194(8)$ & $0.0228(9)$ & $0.0659(15)$ & $0.0055(7)$ & $0.0101(9)$ & $0.0010(9)$ \\
$\mathrm{C} 15$ & $0.0200(9)$ & $0.0268(9)$ & $0.0811(17)$ & $0.0074(7)$ & $0.0050(9)$ & $0.0245(10)$ \\
$\mathrm{C} 16$ & $0.0291(10)$ & $0.0403(11)$ & $0.0527(12)$ & $0.0103(8)$ & $0.0038(9)$ & $0.0296(10)$ \\
$\mathrm{C} 17$ & $0.0266(9)$ & $0.0312(9)$ & $0.0334(9)$ & $0.0122(7)$ & $0.0091(7)$ & $0.0170(8)$ \\
$\mathrm{C} 18$ & $0.0216(7)$ & $0.0187(7)$ & $0.0158(7)$ & $0.0062(6)$ & $0.0057(6)$ & $0.0051(6)$
\end{tabular}


supporting information

\begin{tabular}{lllllll}
$\mathrm{C} 19$ & $0.0323(9)$ & $0.0243(8)$ & $0.0205(8)$ & $0.0114(7)$ & $0.0060(7)$ & $0.0094(6)$ \\
$\mathrm{C} 20$ & $0.0301(9)$ & $0.0345(9)$ & $0.0199(8)$ & $0.0128(7)$ & $-0.0004(7)$ & $0.0083(7)$ \\
$\mathrm{C} 21$ & $0.0227(8)$ & $0.0279(9)$ & $0.0238(8)$ & $0.0040(7)$ & $0.0010(7)$ & $0.0018(7)$ \\
$\mathrm{C} 22$ & $0.0286(9)$ & $0.0223(8)$ & $0.0298(9)$ & $0.0014(7)$ & $0.0014(7)$ & $0.0092(7)$ \\
$\mathrm{C} 23$ & $0.0247(8)$ & $0.0229(8)$ & $0.0222(8)$ & $0.0040(6)$ & $0.0001(6)$ & $0.0101(7)$ \\
$\mathrm{C} 24$ & $0.0516(12)$ & $0.0342(10)$ & $0.0265(9)$ & $0.0102(9)$ & $0.0105(8)$ & $0.0188(8)$ \\
$\mathrm{C} 25$ & $0.0382(11)$ & $0.0231(9)$ & $0.0487(12)$ & $-0.0046(8)$ & $0.0140(9)$ & $0.0083(8)$ \\
$\mathrm{C} 1$ & $0.0340(2)$ & $0.0292(2)$ & $0.0265(2)$ & $0.01592(17)$ & $0.01079(17)$ & $0.00835(17)$ \\
$\mathrm{O} 1 \mathrm{~W}$ & $0.0473(9)$ & $0.0474(9)$ & $0.0704(12)$ & $0.0122(8)$ & $0.0307(9)$ & $0.0117(8)$ \\
\hline
\end{tabular}

Geometric parameters $\left(\AA,{ }^{\circ}\right)$

\begin{tabular}{|c|c|c|c|}
\hline $\mathrm{Fe}-\mathrm{C} 1$ & $2.0239(15)$ & $\mathrm{C} 9-\mathrm{H} 9$ & 0.9500 \\
\hline $\mathrm{Fe}-\mathrm{C} 5$ & $2.0341(16)$ & $\mathrm{C} 10-\mathrm{H} 10$ & 0.9500 \\
\hline $\mathrm{Fe}-\mathrm{C} 10$ & $2.0389(16)$ & C11-H11A & 0.9900 \\
\hline $\mathrm{Fe}-\mathrm{C} 8$ & $2.0411(16)$ & C11-H11B & 0.9900 \\
\hline $\mathrm{Fe}-\mathrm{C} 9$ & $2.0433(16)$ & $\mathrm{C} 12-\mathrm{C} 17$ & $1.386(2)$ \\
\hline $\mathrm{Fe}-\mathrm{C} 2$ & $2.0433(16)$ & $\mathrm{C} 12-\mathrm{C} 13$ & $1.401(2)$ \\
\hline $\mathrm{Fe}-\mathrm{C} 4$ & $2.0469(17)$ & $\mathrm{C} 13-\mathrm{C} 14$ & $1.384(3)$ \\
\hline $\mathrm{Fe}-\mathrm{C} 3$ & $2.0472(16)$ & $\mathrm{C} 13-\mathrm{H} 13$ & 0.9500 \\
\hline $\mathrm{Fe}-\mathrm{C} 6$ & $2.0472(15)$ & $\mathrm{C} 14-\mathrm{C} 15$ & $1.380(3)$ \\
\hline $\mathrm{Fe}-\mathrm{C} 7$ & $2.0489(15)$ & $\mathrm{C} 14-\mathrm{H} 14$ & 0.9500 \\
\hline $\mathrm{P}-\mathrm{C} 6$ & $1.8084(16)$ & $\mathrm{C} 15-\mathrm{C} 16$ & $1.377(3)$ \\
\hline $\mathrm{P}-\mathrm{C} 12$ & $1.8390(17)$ & C15-H15 & 0.9500 \\
\hline $\mathrm{P}-\mathrm{C} 18$ & $1.8427(16)$ & $\mathrm{C} 16-\mathrm{C} 17$ & $1.397(2)$ \\
\hline $\mathrm{N}-\mathrm{C} 24$ & $1.480(2)$ & $\mathrm{C} 16-\mathrm{H} 16$ & 0.9500 \\
\hline $\mathrm{N}-\mathrm{C} 25$ & $1.485(2)$ & C17-H17 & 0.9500 \\
\hline $\mathrm{N}-\mathrm{C} 11$ & $1.508(2)$ & $\mathrm{C} 18-\mathrm{C} 23$ & $1.395(2)$ \\
\hline $\mathrm{N}-\mathrm{H} 1 \mathrm{~N}$ & 0.9207 & $\mathrm{C} 18-\mathrm{C} 19$ & $1.396(2)$ \\
\hline $\mathrm{C} 1-\mathrm{C} 5$ & $1.426(2)$ & $\mathrm{C} 19-\mathrm{C} 20$ & $1.393(2)$ \\
\hline $\mathrm{C} 1-\mathrm{C} 2$ & $1.437(2)$ & C19-H19 & 0.9500 \\
\hline $\mathrm{C} 1-\mathrm{C} 11$ & $1.487(2)$ & $\mathrm{C} 20-\mathrm{C} 21$ & $1.383(3)$ \\
\hline $\mathrm{C} 2-\mathrm{C} 3$ & $1.424(3)$ & $\mathrm{C} 20-\mathrm{H} 20$ & 0.9500 \\
\hline $\mathrm{C} 2-\mathrm{H} 2$ & 0.9500 & $\mathrm{C} 21-\mathrm{C} 22$ & $1.385(2)$ \\
\hline $\mathrm{C} 3-\mathrm{C} 4$ & $1.418(3)$ & $\mathrm{C} 21-\mathrm{H} 21$ & 0.9500 \\
\hline $\mathrm{C} 3-\mathrm{H} 3$ & 0.9500 & $\mathrm{C} 22-\mathrm{C} 23$ & $1.394(2)$ \\
\hline $\mathrm{C} 4-\mathrm{C} 5$ & $1.421(2)$ & $\mathrm{C} 22-\mathrm{H} 22$ & 0.9500 \\
\hline $\mathrm{C} 4-\mathrm{H} 4$ & 0.9500 & $\mathrm{C} 23-\mathrm{H} 23$ & 0.9500 \\
\hline $\mathrm{C} 5-\mathrm{H} 5$ & 0.9500 & $\mathrm{C} 24-\mathrm{H} 24 \mathrm{~A}$ & 0.9800 \\
\hline $\mathrm{C} 6-\mathrm{C} 7$ & $1.428(2)$ & $\mathrm{C} 24-\mathrm{H} 24 \mathrm{~B}$ & 0.9800 \\
\hline $\mathrm{C} 6-\mathrm{C} 10$ & $1.440(2)$ & $\mathrm{C} 24-\mathrm{H} 24 \mathrm{C}$ & 0.9800 \\
\hline $\mathrm{C} 7-\mathrm{C} 8$ & $1.421(2)$ & $\mathrm{C} 25-\mathrm{H} 25 \mathrm{~A}$ & 0.9800 \\
\hline $\mathrm{C} 7-\mathrm{H} 7$ & 0.9500 & $\mathrm{C} 25-\mathrm{H} 25 \mathrm{~B}$ & 0.9800 \\
\hline $\mathrm{C} 8-\mathrm{C} 9$ & $1.420(3)$ & $\mathrm{C} 25-\mathrm{H} 25 \mathrm{C}$ & 0.9800 \\
\hline $\mathrm{C} 8-\mathrm{H} 8$ & 0.9500 & $\mathrm{O} 1 \mathrm{~W}-\mathrm{H} 2 \mathrm{~W}$ & 1.0206 \\
\hline $\mathrm{C} 9-\mathrm{C} 10$ & $1.424(3)$ & $\mathrm{O} 1 \mathrm{~W}-\mathrm{H} 1 \mathrm{~W}$ & 0.9824 \\
\hline $\mathrm{C} 1-\mathrm{Fe}-\mathrm{C} 5$ & $41.14(6)$ & $\mathrm{C} 7-\mathrm{C} 6-\mathrm{P}$ & $128.31(12)$ \\
\hline
\end{tabular}




\begin{tabular}{|c|c|c|c|}
\hline $\mathrm{C} 1-\mathrm{Fe}-\mathrm{C} 10$ & $106.92(7)$ & $\mathrm{C} 10-\mathrm{C} 6-\mathrm{P}$ & $124.39(13)$ \\
\hline $\mathrm{C} 5-\mathrm{Fe}-\mathrm{C} 10$ & $126.23(7)$ & $\mathrm{C} 7-\mathrm{C} 6-\mathrm{Fe}$ & $69.66(9)$ \\
\hline $\mathrm{C} 1-\mathrm{Fe}-\mathrm{C} 8$ & $148.86(7)$ & $\mathrm{C} 10-\mathrm{C} 6-\mathrm{Fe}$ & $69.05(8)$ \\
\hline $\mathrm{C} 5-\mathrm{Fe}-\mathrm{C} 8$ & $115.03(7)$ & $\mathrm{P}-\mathrm{C} 6-\mathrm{Fe}$ & $127.25(8)$ \\
\hline $\mathrm{C} 10-\mathrm{Fe}-\mathrm{C} 8$ & $68.79(7)$ & $\mathrm{C} 8-\mathrm{C} 7-\mathrm{C} 6$ & $108.38(15)$ \\
\hline $\mathrm{C} 1-\mathrm{Fe}-\mathrm{C} 9$ & $115.70(7)$ & $\mathrm{C} 8-\mathrm{C} 7-\mathrm{Fe}$ & $69.38(9)$ \\
\hline $\mathrm{C} 5-\mathrm{Fe}-\mathrm{C} 9$ & $104.86(7)$ & $\mathrm{C} 6-\mathrm{C} 7-\mathrm{Fe}$ & $69.53(8)$ \\
\hline $\mathrm{C} 10-\mathrm{Fe}-\mathrm{C} 9$ & $40.82(7)$ & $\mathrm{C} 8-\mathrm{C} 7-\mathrm{H} 7$ & 125.8 \\
\hline $\mathrm{C} 8-\mathrm{Fe}-\mathrm{C} 9$ & $40.70(8)$ & $\mathrm{C} 6-\mathrm{C} 7-\mathrm{H} 7$ & 125.8 \\
\hline $\mathrm{C} 1-\mathrm{Fe}-\mathrm{C} 2$ & $41.36(6)$ & $\mathrm{Fe}-\mathrm{C} 7-\mathrm{H} 7$ & 126.9 \\
\hline $\mathrm{C} 5-\mathrm{Fe}-\mathrm{C} 2$ & $69.00(7)$ & $\mathrm{C} 9-\mathrm{C} 8-\mathrm{C} 7$ & $108.19(15)$ \\
\hline $\mathrm{C} 10-\mathrm{Fe}-\mathrm{C} 2$ & $119.30(7)$ & $\mathrm{C} 9-\mathrm{C} 8-\mathrm{Fe}$ & $69.73(9)$ \\
\hline $\mathrm{C} 8-\mathrm{Fe}-\mathrm{C} 2$ & $167.52(7)$ & $\mathrm{C} 7-\mathrm{C} 8-\mathrm{Fe}$ & $69.97(9)$ \\
\hline $\mathrm{C} 9-\mathrm{Fe}-\mathrm{C} 2$ & $151.52(8)$ & $\mathrm{C} 9-\mathrm{C} 8-\mathrm{H} 8$ & 125.9 \\
\hline $\mathrm{C} 1-\mathrm{Fe}-\mathrm{C} 4$ & $69.01(7)$ & $\mathrm{C} 7-\mathrm{C} 8-\mathrm{H} 8$ & 125.9 \\
\hline $\mathrm{C} 5-\mathrm{Fe}-\mathrm{C} 4$ & $40.74(7)$ & $\mathrm{Fe}-\mathrm{C} 8-\mathrm{H} 8$ & 126.0 \\
\hline $\mathrm{C} 10-\mathrm{Fe}-\mathrm{C} 4$ & $163.98(7)$ & $\mathrm{C} 8-\mathrm{C} 9-\mathrm{C} 10$ & $108.27(15)$ \\
\hline $\mathrm{C} 8-\mathrm{Fe}-\mathrm{C} 4$ & $106.22(8)$ & $\mathrm{C} 8-\mathrm{C} 9-\mathrm{Fe}$ & $69.57(9)$ \\
\hline $\mathrm{C} 9-\mathrm{Fe}-\mathrm{C} 4$ & $125.71(8)$ & $\mathrm{C} 10-\mathrm{C} 9-\mathrm{Fe}$ & $69.43(9)$ \\
\hline $\mathrm{C} 2-\mathrm{Fe}-\mathrm{C} 4$ & $68.59(7)$ & $\mathrm{C} 8-\mathrm{C} 9-\mathrm{H} 9$ & 125.9 \\
\hline $\mathrm{C} 1-\mathrm{Fe}-\mathrm{C} 3$ & $69.10(6)$ & $\mathrm{C} 10-\mathrm{C} 9-\mathrm{H} 9$ & 125.9 \\
\hline $\mathrm{C} 5-\mathrm{Fe}-\mathrm{C} 3$ & $68.56(7)$ & $\mathrm{Fe}-\mathrm{C} 9-\mathrm{H} 9$ & 126.7 \\
\hline $\mathrm{C} 10-\mathrm{Fe}-\mathrm{C} 3$ & $154.05(8)$ & $\mathrm{C} 9-\mathrm{C} 10-\mathrm{C} 6$ & $107.85(15)$ \\
\hline $\mathrm{C} 8-\mathrm{Fe}-\mathrm{C} 3$ & $128.15(8)$ & $\mathrm{C} 9-\mathrm{C} 10-\mathrm{Fe}$ & $69.75(9)$ \\
\hline $\mathrm{C} 9-\mathrm{Fe}-\mathrm{C} 3$ & $164.68(8)$ & $\mathrm{C} 6-\mathrm{C} 10-\mathrm{Fe}$ & $69.67(9)$ \\
\hline $\mathrm{C} 2-\mathrm{Fe}-\mathrm{C} 3$ & $40.75(7)$ & $\mathrm{C} 9-\mathrm{C} 10-\mathrm{H} 10$ & 126.1 \\
\hline $\mathrm{C} 4-\mathrm{Fe}-\mathrm{C} 3$ & $40.52(8)$ & $\mathrm{C} 6-\mathrm{C} 10-\mathrm{H} 10$ & 126.1 \\
\hline $\mathrm{C} 1-\mathrm{Fe}-\mathrm{C} 6$ & $129.28(6)$ & $\mathrm{Fe}-\mathrm{C} 10-\mathrm{H} 10$ & 126.1 \\
\hline $\mathrm{C} 5-\mathrm{Fe}-\mathrm{C} 6$ & $166.26(7)$ & $\mathrm{C} 1-\mathrm{C} 11-\mathrm{N}$ & $112.09(13)$ \\
\hline $\mathrm{C} 10-\mathrm{Fe}-\mathrm{C} 6$ & $41.28(6)$ & $\mathrm{C} 1-\mathrm{C} 11-\mathrm{H} 11 \mathrm{~A}$ & 109.2 \\
\hline $\mathrm{C} 8-\mathrm{Fe}-\mathrm{C} 6$ & $68.83(7)$ & $\mathrm{N}-\mathrm{C} 11-\mathrm{H} 11 \mathrm{~A}$ & 109.2 \\
\hline $\mathrm{C} 9-\mathrm{Fe}-\mathrm{C} 6$ & $68.93(6)$ & $\mathrm{C} 1-\mathrm{C} 11-\mathrm{H} 11 \mathrm{~B}$ & 109.2 \\
\hline $\mathrm{C} 2-\mathrm{Fe}-\mathrm{C} 6$ & $110.24(7)$ & $\mathrm{N}-\mathrm{C} 11-\mathrm{H} 11 \mathrm{~B}$ & 109.2 \\
\hline $\mathrm{C} 4-\mathrm{Fe}-\mathrm{C} 6$ & $152.74(7)$ & $\mathrm{H} 11 \mathrm{~A}-\mathrm{C} 11-\mathrm{H} 11 \mathrm{~B}$ & 107.9 \\
\hline $\mathrm{C} 3-\mathrm{Fe}-\mathrm{C} 6$ & $120.44(7)$ & $\mathrm{C} 17-\mathrm{C} 12-\mathrm{C} 13$ & $118.37(16)$ \\
\hline $\mathrm{C} 1-\mathrm{Fe}-\mathrm{C} 7$ & $168.72(6)$ & $\mathrm{C} 17-\mathrm{C} 12-\mathrm{P}$ & $123.83(13)$ \\
\hline $\mathrm{C} 5-\mathrm{Fe}-\mathrm{C} 7$ & $149.81(7)$ & $\mathrm{C} 13-\mathrm{C} 12-\mathrm{P}$ & $117.78(14)$ \\
\hline $\mathrm{C} 10-\mathrm{Fe}-\mathrm{C} 7$ & $68.83(7)$ & $\mathrm{C} 14-\mathrm{C} 13-\mathrm{C} 12$ & $120.41(19)$ \\
\hline $\mathrm{C} 8-\mathrm{Fe}-\mathrm{C} 7$ & $40.66(6)$ & $\mathrm{C} 14-\mathrm{C} 13-\mathrm{H} 13$ & 119.8 \\
\hline $\mathrm{C} 9-\mathrm{Fe}-\mathrm{C} 7$ & $68.44(7)$ & $\mathrm{C} 12-\mathrm{C} 13-\mathrm{H} 13$ & 119.8 \\
\hline $\mathrm{C} 2-\mathrm{Fe}-\mathrm{C} 7$ & $130.66(6)$ & $\mathrm{C} 15-\mathrm{C} 14-\mathrm{C} 13$ & $120.62(19)$ \\
\hline $\mathrm{C} 4-\mathrm{Fe}-\mathrm{C} 7$ & $117.98(7)$ & $\mathrm{C} 15-\mathrm{C} 14-\mathrm{H} 14$ & 119.7 \\
\hline $\mathrm{C} 3-\mathrm{Fe}-\mathrm{C} 7$ & $109.84(7)$ & $\mathrm{C} 13-\mathrm{C} 14-\mathrm{H} 14$ & 119.7 \\
\hline $\mathrm{C} 6-\mathrm{Fe}-\mathrm{C} 7$ & $40.81(6)$ & $\mathrm{C} 16-\mathrm{C} 15-\mathrm{C} 14$ & $119.70(18)$ \\
\hline $\mathrm{C} 6-\mathrm{P}-\mathrm{C} 12$ & $100.79(7)$ & $\mathrm{C} 16-\mathrm{C} 15-\mathrm{H} 15$ & 120.1 \\
\hline $\mathrm{C} 6-\mathrm{P}-\mathrm{C} 18$ & $101.23(7)$ & $\mathrm{C} 14-\mathrm{C} 15-\mathrm{H} 15$ & 120.1 \\
\hline $\mathrm{C} 12-\mathrm{P}-\mathrm{C} 18$ & $100.82(7)$ & $\mathrm{C} 15-\mathrm{C} 16-\mathrm{C} 17$ & $120.0(2)$ \\
\hline $\mathrm{C} 24-\mathrm{N}-\mathrm{C} 25$ & $111.96(15)$ & $\mathrm{C} 15-\mathrm{C} 16-\mathrm{H} 16$ & 120.0 \\
\hline
\end{tabular}




\begin{tabular}{|c|c|c|c|}
\hline $\mathrm{C} 24-\mathrm{N}-\mathrm{C} 11$ & $112.50(13)$ & $\mathrm{C} 17-\mathrm{C} 16-\mathrm{H} 16$ & 120.0 \\
\hline $\mathrm{C} 25-\mathrm{N}-\mathrm{C} 11$ & $110.50(14)$ & $\mathrm{C} 12-\mathrm{C} 17-\mathrm{C} 16$ & $120.80(18)$ \\
\hline $\mathrm{C} 24-\mathrm{N}-\mathrm{H} 1 \mathrm{~N}$ & 105.9 & $\mathrm{C} 12-\mathrm{C} 17-\mathrm{H} 17$ & 119.6 \\
\hline $\mathrm{C} 25-\mathrm{N}-\mathrm{H} 1 \mathrm{~N}$ & 107.8 & $\mathrm{C} 16-\mathrm{C} 17-\mathrm{H} 17$ & 119.6 \\
\hline $\mathrm{C} 11-\mathrm{N}-\mathrm{H} 1 \mathrm{~N}$ & 107.9 & $\mathrm{C} 23-\mathrm{C} 18-\mathrm{C} 19$ & $118.43(15)$ \\
\hline $\mathrm{C} 5-\mathrm{C} 1-\mathrm{C} 2$ & $107.59(15)$ & $\mathrm{C} 23-\mathrm{C} 18-\mathrm{P}$ & $123.76(12)$ \\
\hline $\mathrm{C} 5-\mathrm{C} 1-\mathrm{C} 11$ & $124.99(15)$ & $\mathrm{C} 19-\mathrm{C} 18-\mathrm{P}$ & $117.78(12)$ \\
\hline $\mathrm{C} 2-\mathrm{C} 1-\mathrm{C} 11$ & $127.30(15)$ & $\mathrm{C} 20-\mathrm{C} 19-\mathrm{C} 18$ & $120.71(16)$ \\
\hline $\mathrm{C} 5-\mathrm{C} 1-\mathrm{Fe}$ & $69.82(9)$ & $\mathrm{C} 20-\mathrm{C} 19-\mathrm{H} 19$ & 119.6 \\
\hline $\mathrm{C} 2-\mathrm{C} 1-\mathrm{Fe}$ & $70.04(9)$ & $\mathrm{C} 18-\mathrm{C} 19-\mathrm{H} 19$ & 119.6 \\
\hline $\mathrm{C} 11-\mathrm{C} 1-\mathrm{Fe}$ & $122.55(11)$ & $\mathrm{C} 21-\mathrm{C} 20-\mathrm{C} 19$ & $120.27(16)$ \\
\hline $\mathrm{C} 3-\mathrm{C} 2-\mathrm{C} 1$ & $107.63(15)$ & $\mathrm{C} 21-\mathrm{C} 20-\mathrm{H} 20$ & 119.9 \\
\hline $\mathrm{C} 3-\mathrm{C} 2-\mathrm{Fe}$ & $69.77(9)$ & $\mathrm{C} 19-\mathrm{C} 20-\mathrm{H} 20$ & 119.9 \\
\hline $\mathrm{C} 1-\mathrm{C} 2-\mathrm{Fe}$ & $68.59(9)$ & $\mathrm{C} 20-\mathrm{C} 21-\mathrm{C} 22$ & $119.64(16)$ \\
\hline $\mathrm{C} 3-\mathrm{C} 2-\mathrm{H} 2$ & 126.2 & $\mathrm{C} 20-\mathrm{C} 21-\mathrm{H} 21$ & 120.2 \\
\hline $\mathrm{C} 1-\mathrm{C} 2-\mathrm{H} 2$ & 126.2 & $\mathrm{C} 22-\mathrm{C} 21-\mathrm{H} 21$ & 120.2 \\
\hline $\mathrm{Fe}-\mathrm{C} 2-\mathrm{H} 2$ & 127.0 & $\mathrm{C} 21-\mathrm{C} 22-\mathrm{C} 23$ & $120.31(16)$ \\
\hline $\mathrm{C} 4-\mathrm{C} 3-\mathrm{C} 2$ & $108.37(15)$ & $\mathrm{C} 21-\mathrm{C} 22-\mathrm{H} 22$ & 119.8 \\
\hline $\mathrm{C} 4-\mathrm{C} 3-\mathrm{Fe}$ & $69.73(10)$ & $\mathrm{C} 23-\mathrm{C} 22-\mathrm{H} 22$ & 119.8 \\
\hline $\mathrm{C} 2-\mathrm{C} 3-\mathrm{Fe}$ & $69.48(9)$ & $\mathrm{C} 22-\mathrm{C} 23-\mathrm{C} 18$ & $120.63(15)$ \\
\hline $\mathrm{C} 4-\mathrm{C} 3-\mathrm{H} 3$ & 125.8 & $\mathrm{C} 22-\mathrm{C} 23-\mathrm{H} 23$ & 119.7 \\
\hline $\mathrm{C} 2-\mathrm{C} 3-\mathrm{H} 3$ & 125.8 & $\mathrm{C} 18-\mathrm{C} 23-\mathrm{H} 23$ & 119.7 \\
\hline $\mathrm{Fe}-\mathrm{C} 3-\mathrm{H} 3$ & 126.6 & $\mathrm{~N}-\mathrm{C} 24-\mathrm{H} 24 \mathrm{~A}$ & 109.5 \\
\hline $\mathrm{C} 3-\mathrm{C} 4-\mathrm{C} 5$ & $108.16(16)$ & $\mathrm{N}-\mathrm{C} 24-\mathrm{H} 24 \mathrm{~B}$ & 109.5 \\
\hline $\mathrm{C} 3-\mathrm{C} 4-\mathrm{Fe}$ & $69.75(10)$ & $\mathrm{H} 24 \mathrm{~A}-\mathrm{C} 24-\mathrm{H} 24 \mathrm{~B}$ & 109.5 \\
\hline $\mathrm{C} 5-\mathrm{C} 4-\mathrm{Fe}$ & $69.14(9)$ & $\mathrm{N}-\mathrm{C} 24-\mathrm{H} 24 \mathrm{C}$ & 109.5 \\
\hline $\mathrm{C} 3-\mathrm{C} 4-\mathrm{H} 4$ & 125.9 & $\mathrm{H} 24 \mathrm{~A}-\mathrm{C} 24-\mathrm{H} 24 \mathrm{C}$ & 109.5 \\
\hline $\mathrm{C} 5-\mathrm{C} 4-\mathrm{H} 4$ & 125.9 & $\mathrm{H} 24 \mathrm{~B}-\mathrm{C} 24-\mathrm{H} 24 \mathrm{C}$ & 109.5 \\
\hline $\mathrm{Fe}-\mathrm{C} 4-\mathrm{H} 4$ & 126.8 & $\mathrm{~N}-\mathrm{C} 25-\mathrm{H} 25 \mathrm{~A}$ & 109.5 \\
\hline $\mathrm{C} 4-\mathrm{C} 5-\mathrm{C} 1$ & $108.24(15)$ & $\mathrm{N}-\mathrm{C} 25-\mathrm{H} 25 \mathrm{~B}$ & 109.5 \\
\hline $\mathrm{C} 4-\mathrm{C} 5-\mathrm{Fe}$ & $70.11(10)$ & $\mathrm{H} 25 \mathrm{~A}-\mathrm{C} 25-\mathrm{H} 25 \mathrm{~B}$ & 109.5 \\
\hline $\mathrm{C} 1-\mathrm{C} 5-\mathrm{Fe}$ & $69.05(9)$ & $\mathrm{N}-\mathrm{C} 25-\mathrm{H} 25 \mathrm{C}$ & 109.5 \\
\hline $\mathrm{C} 4-\mathrm{C} 5-\mathrm{H} 5$ & 125.9 & $\mathrm{H} 25 \mathrm{~A}-\mathrm{C} 25-\mathrm{H} 25 \mathrm{C}$ & 109.5 \\
\hline $\mathrm{C} 1-\mathrm{C} 5-\mathrm{H} 5$ & 125.9 & $\mathrm{H} 25 \mathrm{~B}-\mathrm{C} 25-\mathrm{H} 25 \mathrm{C}$ & 109.5 \\
\hline $\mathrm{Fe}-\mathrm{C} 5-\mathrm{H} 5$ & 126.5 & $\mathrm{H} 2 \mathrm{~W}-\mathrm{O} 1 \mathrm{~W}-\mathrm{H} 1 \mathrm{~W}$ & 107.9 \\
\hline $\mathrm{C} 7-\mathrm{C} 6-\mathrm{C} 10$ & $107.30(14)$ & & \\
\hline $\mathrm{C} 5-\mathrm{C} 1-\mathrm{C} 2-\mathrm{C} 3$ & $-0.93(17)$ & $\mathrm{Fe}-\mathrm{C} 9-\mathrm{C} 10-\mathrm{C} 6$ & $-59.48(10)$ \\
\hline $\mathrm{C} 11-\mathrm{C} 1-\mathrm{C} 2-\mathrm{C} 3$ & $175.34(14)$ & $\mathrm{C} 8-\mathrm{C} 9-\mathrm{C} 10-\mathrm{Fe}$ & $58.89(11)$ \\
\hline $\mathrm{Fe}-\mathrm{C} 1-\mathrm{C} 2-\mathrm{C} 3$ & $59.05(11)$ & $\mathrm{C} 7-\mathrm{C} 6-\mathrm{C} 10-\mathrm{C} 9$ & $0.14(17)$ \\
\hline $\mathrm{C} 5-\mathrm{C} 1-\mathrm{C} 2-\mathrm{Fe}$ & $-59.99(11)$ & $\mathrm{P}-\mathrm{C} 6-\mathrm{C} 10-\mathrm{C} 9$ & $-178.91(11)$ \\
\hline $\mathrm{C} 11-\mathrm{C} 1-\mathrm{C} 2-\mathrm{Fe}$ & $116.29(15)$ & $\mathrm{Fe}-\mathrm{C} 6-\mathrm{C} 10-\mathrm{C} 9$ & $59.53(11)$ \\
\hline $\mathrm{C} 1-\mathrm{C} 2-\mathrm{C} 3-\mathrm{C} 4$ & $0.75(18)$ & $\mathrm{C} 7-\mathrm{C} 6-\mathrm{C} 10-\mathrm{Fe}$ & $-59.39(10)$ \\
\hline $\mathrm{Fe}-\mathrm{C} 2-\mathrm{C} 3-\mathrm{C} 4$ & $59.06(12)$ & $\mathrm{P}-\mathrm{C} 6-\mathrm{C} 10-\mathrm{Fe}$ & $121.56(11)$ \\
\hline $\mathrm{C} 1-\mathrm{C} 2-\mathrm{C} 3-\mathrm{Fe}$ & $-58.32(10)$ & $\mathrm{C} 5-\mathrm{C} 1-\mathrm{C} 11-\mathrm{N}$ & $-77.55(19)$ \\
\hline $\mathrm{C} 2-\mathrm{C} 3-\mathrm{C} 4-\mathrm{C} 5$ & $-0.27(19)$ & $\mathrm{C} 2-\mathrm{C} 1-\mathrm{C} 11-\mathrm{N}$ & $106.78(17)$ \\
\hline $\mathrm{Fe}-\mathrm{C} 3-\mathrm{C} 4-\mathrm{C} 5$ & $58.64(11)$ & $\mathrm{Fe}-\mathrm{C} 1-\mathrm{C} 11-\mathrm{N}$ & $-164.53(10)$ \\
\hline $\mathrm{C} 2-\mathrm{C} 3-\mathrm{C} 4-\mathrm{Fe}$ & $-58.91(11)$ & $\mathrm{C} 24-\mathrm{N}-\mathrm{C} 11-\mathrm{C} 1$ & $-59.33(18)$ \\
\hline
\end{tabular}




\begin{tabular}{|c|c|c|c|}
\hline $\mathrm{C} 3-\mathrm{C} 4-\mathrm{C} 5-\mathrm{C} 1$ & $-0.31(18)$ & $\mathrm{C} 25-\mathrm{N}-\mathrm{C} 11-\mathrm{C} 1$ & $174.72(14)$ \\
\hline $\mathrm{Fe}-\mathrm{C} 4-\mathrm{C} 5-\mathrm{C} 1$ & $58.70(11)$ & $\mathrm{C} 6-\mathrm{P}-\mathrm{C} 12-\mathrm{C} 17$ & $-3.86(16)$ \\
\hline $\mathrm{C} 3-\mathrm{C} 4-\mathrm{C} 5-\mathrm{Fe}$ & $-59.01(12)$ & $\mathrm{C} 18-\mathrm{P}-\mathrm{C} 12-\mathrm{C} 17$ & $-107.64(15)$ \\
\hline $\mathrm{C} 2-\mathrm{C} 1-\mathrm{C} 5-\mathrm{C} 4$ & $0.77(17)$ & $\mathrm{C} 6-\mathrm{P}-\mathrm{C} 12-\mathrm{C} 13$ & $177.80(13)$ \\
\hline $\mathrm{C} 11-\mathrm{C} 1-\mathrm{C} 5-\mathrm{C} 4$ & $-175.61(14)$ & $\mathrm{C} 18-\mathrm{P}-\mathrm{C} 12-\mathrm{C} 13$ & $74.02(14)$ \\
\hline $\mathrm{Fe}-\mathrm{C} 1-\mathrm{C} 5-\mathrm{C} 4$ & $-59.36(11)$ & $\mathrm{C} 17-\mathrm{C} 12-\mathrm{C} 13-\mathrm{C} 14$ & $2.5(2)$ \\
\hline $\mathrm{C} 2-\mathrm{C} 1-\mathrm{C} 5-\mathrm{Fe}$ & $60.13(10)$ & $\mathrm{P}-\mathrm{C} 12-\mathrm{C} 13-\mathrm{C} 14$ & $-179.02(14)$ \\
\hline $\mathrm{C} 11-\mathrm{C} 1-\mathrm{C} 5-\mathrm{Fe}$ & $-116.26(15)$ & $\mathrm{C} 12-\mathrm{C} 13-\mathrm{C} 14-\mathrm{C} 15$ & $-2.5(3)$ \\
\hline $\mathrm{C} 12-\mathrm{P}-\mathrm{C} 6-\mathrm{C} 7$ & $-89.13(14)$ & $\mathrm{C} 13-\mathrm{C} 14-\mathrm{C} 15-\mathrm{C} 16$ & $0.6(3)$ \\
\hline $\mathrm{C} 18-\mathrm{P}-\mathrm{C} 6-\mathrm{C} 7$ & $14.33(15)$ & $\mathrm{C} 14-\mathrm{C} 15-\mathrm{C} 16-\mathrm{C} 17$ & $1.2(3)$ \\
\hline $\mathrm{C} 12-\mathrm{P}-\mathrm{C} 6-\mathrm{C} 10$ & $89.71(13)$ & $\mathrm{C} 13-\mathrm{C} 12-\mathrm{C} 17-\mathrm{C} 16$ & $-0.8(3)$ \\
\hline $\mathrm{C} 18-\mathrm{P}-\mathrm{C} 6-\mathrm{C} 10$ & $-166.83(13)$ & $\mathrm{P}-\mathrm{C} 12-\mathrm{C} 17-\mathrm{C} 16$ & $-179.14(14)$ \\
\hline $\mathrm{C} 12-\mathrm{P}-\mathrm{C} 6-\mathrm{Fe}$ & $178.35(9)$ & $\mathrm{C} 15-\mathrm{C} 16-\mathrm{C} 17-\mathrm{C} 12$ & $-1.0(3)$ \\
\hline $\mathrm{C} 18-\mathrm{P}-\mathrm{C} 6-\mathrm{Fe}$ & $-78.20(11)$ & $\mathrm{C} 6-\mathrm{P}-\mathrm{C} 18-\mathrm{C} 23$ & $-83.71(15)$ \\
\hline $\mathrm{C} 10-\mathrm{C} 6-\mathrm{C} 7-\mathrm{C} 8$ & $0.36(17)$ & $\mathrm{C} 12-\mathrm{P}-\mathrm{C} 18-\mathrm{C} 23$ & $19.72(15)$ \\
\hline $\mathrm{P}-\mathrm{C} 6-\mathrm{C} 7-\mathrm{C} 8$ & $179.36(12)$ & $\mathrm{C} 6-\mathrm{P}-\mathrm{C} 18-\mathrm{C} 19$ & $97.97(13)$ \\
\hline $\mathrm{Fe}-\mathrm{C} 6-\mathrm{C} 7-\mathrm{C} 8$ & $-58.65(11)$ & $\mathrm{C} 12-\mathrm{P}-\mathrm{C} 18-\mathrm{C} 19$ & $-158.60(13)$ \\
\hline $\mathrm{C} 10-\mathrm{C} 6-\mathrm{C} 7-\mathrm{Fe}$ & $59.01(10)$ & $\mathrm{C} 23-\mathrm{C} 18-\mathrm{C} 19-\mathrm{C} 20$ & $-0.1(2)$ \\
\hline $\mathrm{P}-\mathrm{C} 6-\mathrm{C} 7-\mathrm{Fe}$ & $-121.99(12)$ & $\mathrm{P}-\mathrm{C} 18-\mathrm{C} 19-\mathrm{C} 20$ & $178.34(13)$ \\
\hline $\mathrm{C} 6-\mathrm{C} 7-\mathrm{C} 8-\mathrm{C} 9$ & $-0.73(18)$ & $\mathrm{C} 18-\mathrm{C} 19-\mathrm{C} 20-\mathrm{C} 21$ & $-0.7(3)$ \\
\hline $\mathrm{Fe}-\mathrm{C} 7-\mathrm{C} 8-\mathrm{C} 9$ & $-59.47(11)$ & $\mathrm{C} 19-\mathrm{C} 20-\mathrm{C} 21-\mathrm{C} 22$ & $0.7(3)$ \\
\hline $\mathrm{C} 6-\mathrm{C} 7-\mathrm{C} 8-\mathrm{Fe}$ & $58.75(11)$ & $\mathrm{C} 20-\mathrm{C} 21-\mathrm{C} 22-\mathrm{C} 23$ & $0.1(3)$ \\
\hline $\mathrm{C} 7-\mathrm{C} 8-\mathrm{C} 9-\mathrm{C} 10$ & $0.81(18)$ & $\mathrm{C} 21-\mathrm{C} 22-\mathrm{C} 23-\mathrm{C} 18$ & $-0.8(3)$ \\
\hline $\mathrm{Fe}-\mathrm{C} 8-\mathrm{C} 9-\mathrm{C} 10$ & $-58.81(11)$ & $\mathrm{C} 19-\mathrm{C} 18-\mathrm{C} 23-\mathrm{C} 22$ & $0.8(3)$ \\
\hline $\mathrm{C} 7-\mathrm{C} 8-\mathrm{C} 9-\mathrm{Fe}$ & $59.62(11)$ & $\mathrm{P}-\mathrm{C} 18-\mathrm{C} 23-\mathrm{C} 22$ & $-177.51(14)$ \\
\hline $\mathrm{C} 8-\mathrm{C} 9-\mathrm{C} 10-\mathrm{C} 6$ & $-0.58(18)$ & & \\
\hline
\end{tabular}

Hydrogen-bond geometry $\left(\AA,{ }^{\circ}\right)$

\begin{tabular}{lllll}
\hline$D-\mathrm{H} \cdots A$ & $D-\mathrm{H}$ & $\mathrm{H} \cdots A$ & $D \cdots A$ & $D-\mathrm{H} \cdots A$ \\
\hline $\mathrm{N}-\mathrm{H} 1 N \cdots \mathrm{Cl}$ & 0.92 & 2.13 & $3.0323(16)$ & 167 \\
$\mathrm{O} 1 W-\mathrm{H} 1 W \cdots \mathrm{Cl}$ & 0.98 & 2.23 & $3.2162(19)$ & 177 \\
$\mathrm{O} 1 W-\mathrm{H} 2 W \cdots \mathrm{Cl}$ & 1.02 & 2.29 & $3.289(2)$ & 166 \\
$\mathrm{C} 10-\mathrm{H} 10 \cdots \mathrm{O} 1 W^{\mathrm{i}}$ & 0.95 & 2.46 & $3.390(3)$ & 165 \\
$\mathrm{C} 11-\mathrm{H} 11 B \cdots \mathrm{Cl}^{\mathrm{ii}}$ & 0.99 & 2.77 & $3.7369(17)$ & 167 \\
\hline
\end{tabular}

Symmetry codes: (i) $-x,-y+2,-z+2$; (ii) $-x+1,-y+2,-z+2$. 